\title{
The standard candle aspect of carbon stars
}

\author{
P. Battinelli ${ }^{1}$ and S. Demers ${ }^{2}$
}

1 INAF, Osservatorio Astronomico di Roma, Viale del Parco Mellini 84, 00136 Roma, Italia e-mail: battinel@oarhp1.rm.astro.it

2 Département de Physique, Université de Montréal, CP 6128, succursale centre-ville, Montréal, Québec H3C 3J7, Canada e-mail: demers@astro.umontreal.ca

Received 3 May 2005 / Accepted 3 July 2005

\section{ABSTRACT}

This paper represents the second part of our effort to summarise various aspects of our homogeneous survey of carbon stars in nearby galaxies. Here we investigate the relationships between the size of the $\mathrm{C}$ star populations and the luminosity of the parent galaxies. We also explore the constancy of $\left\langle M_{I}\right\rangle$ for various metallicities and properties of the parent galaxies. We conclude that $\mathrm{C}$ stars are a viable standard candle for galaxies large enough to contain one hundred or more $\mathrm{C}$ stars. The application of narrow-band filters limits, however, the use of $\mathrm{C}$ stars as standard candles to no more than $\sim 2 \mathrm{Mpc}$ with currently available ground-based telescopes. Near-IR photometry from space observations can significantly push the limit of detectability of $\mathrm{C}$ stars thus making them an interesting alternative to the TRGB method.

Key words. stars: carbon - stars: AGB and post-AGB - cosmology: distance scale

\section{Introduction}

The spectral type approach to classify $\mathrm{C}$ and M stars, pursued in the 1970's toward the Magellanic Clouds could not reach much farther away. It was replaced, in the nineteen eighties, by a photometric technique based on two (or three) narrow band filters (Palmer \& Wing 1982; Richer et al. 1984; Cook et al. 1986). Following a hiatus of more than ten years, this $(\mathrm{CN}-\mathrm{TiO})$ approach has been recently exploited to survey a number of Local Group members with the aim of better defining the properties of $\mathrm{C}$ stars in various galaxies.

Richer et al. (1985) were the first to use the mean apparent magnitude of their newly discovered C stars in NGC 205 to obtain a distance estimate for this galaxy. They noted that the $\mathrm{C}$ stars in the two Magellanic Clouds have essentially the same mean absolute magnitude and, on the assumption that the C stars of NGC 205 have the same mean magnitude, could deduce its distance.

A few years later, Cook et al. (1986) publish the results of their survey of half a dozen galaxies and put the question into a more general base, i.e. the possibility of a universal luminosity function for $\mathrm{C}$ stars. They acknowledge that their current small number statistics is preventing a definite answer. Indeed, these early 1980's studies, even though using the best telescopes available at the time, are based on data acquired with small format CCDs and thus only small fields in each galaxy were observed leading to small number statistics.

The pursuit of the standard candle approach culminated in the second half of the 1980's with a series of papers by Richer and collaborators who reach the Sculptor Group galaxies (Richer et al. 1985; Pritchet et al. 1987). The standard candle aspect of C stars was applied to the NGC 300 observations by Richer et al. (1985). They enumerate the advantages (still valid today) offered by $\mathrm{C}$ stars: 1 ) $\mathrm{C}$ stars are the brightest giants in the $I$ band; 2) their $I$ magnitudes show a small dispersion; 3 ) they are easy to identify and cannot be confused with Galactic objects; 4) more importantly, they are simpler to use than Cepheids because no follow-up observations are needed to establish periods. The $\mathrm{C}$ star approach to distance determination was then forgotten for nearly twenty years while a different powerful method was nearly universally accepted.

Throughout the 1980's observers used the apparent magnitude of the brightest red giants, seen in a galaxy, to estimate the distance of that galaxy. During this decade the Tip of the Red Giant Branch (TRGB) method to determine distance evolved from simply an estimate of magnitudes (see for example Graham 1982) to a fully developed technique described by Lee et al. (1993). Since that time, the technique has been intensively used to determine distances of literally hundreds of galaxies in our surroundings. For an updated assessment of the TRGB method see Bellazzini et al. (2001). The success of this method diverted the attention from the $\mathrm{C}$ stars as standard candles.

At about the same time, the planetary nebulae became a tool for distance scale (see Jacoby et al. 1992 for a review of this method). Distances of galaxies could be determined from the luminosity function in the light of [OIII] $\lambda 5007$. The luminosity function was calibrated with Local Group galaxies and distances were measured up to several Mpc. This method implies that both the shape of the luminosity function and the magnitude of the brightest PN are universal. Distances are obtained 
Table 1. Cool C star populations in nearby galaxy.

\begin{tabular}{|c|c|c|c|c|c|c|c|}
\hline Galaxy & $M_{V}$ & {$[\mathrm{Fe} / \mathrm{H}]$} & $N_{\mathrm{c}}$ & $\left\langle M_{I}\right\rangle$ & $\left\langle(R-I)_{0}\right\rangle$ & $C / M 0+$ & $\left\langle M_{\mathrm{bol}}\right\rangle$ \\
\hline WLM & -14.6 & -1.4 & 147 & -4.69 & 1.103 & $15.0 \pm 7.1$ & -4.91 \\
\hline IC $10 \dagger$ & -15.6 & -0.8 & 684 & - & 1.112 & $0.23 \pm 0.02$ & - \\
\hline NGC 147 & -15.6 & -1.0 & 297 & -4.42 & 1.117 & $0.30 \pm 0.03$ & -4.66 \\
\hline NGC 185 & -15.6 & -1.3 & 162 & -4.48 & 1.108 & $0.24 \pm 0.03$ & -4.71 \\
\hline NGC 205 & -16.7 & -0.85 & 498 & -4.53 & 1.131 & $0.20 \pm 0.01$ & -4.80 \\
\hline M 31\% & -21.1 & -0.7 & (780) & -4.55 & 1.181 & $0.08 \pm 0.004$ & -4.88 \\
\hline IC 1613 & -14.8 & -1.2 & 173 & -4.76 & 1.176 & $1.48 \pm 0.31$ & -5.08 \\
\hline NGC 3109 & -15.7 & -1.7 & 421 & -4.77 & 1.163 & $1.85 \pm 0.21$ & -5.07 \\
\hline Leo I & -12.1 & -1.5 & 13 & -4.68 & 1.061 & $6.50 \pm 6.40$ & -4.85 \\
\hline Sgr DIG & -11.0 & -2.1 & 16 & -4.96 & 1.083 & $4.0 \pm 3.0$ & -5.16 \\
\hline NGC 6822 & -15.0 & -1.25 & 927 & -4.57 & 1.190 & $0.90 \pm 0.08$ & -4.91 \\
\hline DDO 210 & -10.3 & -1.9 & 2 & -4.91 & 1.007 & $0.09 \pm 0.08$ & -5.01 \\
\hline Pegasus & -13.1 & -1.1 & 26 & -4.68 & 1.084 & $0.62 \pm 0.22$ & -4.88 \\
\hline LMC & -18.5 & -0.5 & $\sim 8000$ & -4.58 & 1.12 & & \\
\hline SMC & -17.1 & -0.8 & $\sim 2000$ & -4.55 & & & \\
\hline M 33 & -18.9 & -1.0 & $\sim 10000$ & -4.57 & 1.42 & & \\
\hline DDO 190 & -14.5 & -1.55 & $\sim 100$ & -4.71 & 1.10 & 2.5 & -4.98 \\
\hline AndII & -11.2 & -1.5 & 7 & -4.33 & 1.20 & & -4.47 \\
\hline AndVII & -12.0 & -1.5 & 3 & -3.68 & & & \\
\hline Fornax & -13.1 & -1.2 & 26 & & & & \\
\hline
\end{tabular}

$\dagger$ The distance modulus for IC 10 was obtained by Demers et al. (2004) from the average $I$-luminosity of C stars. $\ddagger$ The number of C stars refers to the SW1 field (see Battinelli et al. 2003).

fitting the observed high-luminosity tail of the model luminosity function. As our observations of IC 10 have demonstrated, $\mathrm{C}$ stars are at least one order of magnitude more numerous than PNe. The numbers of PNe recently discovered in NGC 147 and NGC 185 by Corradi et al. (2005) represent barely a few percent the numbers of $\mathrm{C}$ stars.

Today, we feel that the $\mathrm{C}$ stars present advantages not fully realised twenty years ago, so that they can be used to complement the TRGB technique in some cases. Briefly, we note that the TRGB is at $M_{I}=-4.04$ (Bellazzini et al. 2001) while $\left\langle M_{I}\right\rangle=-4.58$ for $\mathrm{C}$ stars. In the near infrared this magnitude difference is even more pronounced and $\mathrm{C}$ stars can be easily identify form their $(J-K)$ colours (Nikolaev \& Weinberg 2000). One of the major uncertainties in the determination of the luminosity of the TRGB is the combined effect of blending and AGB stars above the tip that may lead to an overestimate of the luminosity. Such effect is clearly not important for C stars since they are the brightest red objects in galaxies and are identified from the colour-colour diagram. $\mathrm{C}$ stars are obviously less numerous than stars at the TRGB but, as the use of large format CCDs and mosaics has proved (see e.g. Letarte et al. 2002; Demers et al. 2004), C stars can be identify in large numbers well into the low density peripheries of galaxies.

We recently compile a summary of our survey of Local Group galaxies, (Battinelli \& Demers 2005a, hereafter Paper I), these data can now be used to demonstrate the usefulness of
C stars, not only as standard candle but also to learn about the properties of the intermediate-age population in nearby galaxies.

\section{The sample of extragalactic $C$ stars}

In Paper I we discussed the importance of dealing exclusively with homogeneous $\mathrm{C}$ star samples since any differences in the observational methods or in the identification criteria affect the "C star" properties making the comparison among different galaxies difficult. For example, the definition of $\mathrm{C}$ stars adopted by Margon et al. (2002) to identify high latitude $\mathrm{C}$ stars is quite different from ours because theirs accepts fainter bluer $\mathrm{C}$ stars. For these reasons, the summary of the currently available data, taken from Paper I and given in Table 1 includes mostly our own surveys plus a few other galaxies for which the $\mathrm{C}$ stars were acquired following our criteria or criteria easy to compare. We present below details on these newly added galaxies. It is important to stress that our definition of $\mathrm{C}$ stars includes only stars redder than $(R-I)_{0}=0.90$ (or the corresponding colours in other systems) which represents the colour of the M0 spectral-type stars as explained in Paper I.

Large Magellanic Cloud: we use the $I, R$ observations of Costa \& Frogel (1996), selecting stars with $(R-I)_{0}>0.90$. We adopt a distance modulus of 18.50 and an abundance of its older population, $[\mathrm{Fe} / \mathrm{H}]=-0.5$. The number of $\mathrm{C}$ stars corresponds 
to an approximation of recent estimates, see Cioni \& Habing (2003).

Small Magellanic Cloud: we use the DENIS data of C stars with $(J-K)>1.4$ which was shown by Demers et al. (2002) to be equivalent to our $(R-I)$ criterion, for which $\langle I\rangle=14.40$. We adopt the canonical abundance $[\mathrm{Fe} / \mathrm{H}]=-0.8$. The number of C stars is believed to be 2000 , see Cioni \& Habing (2003) for discussion.

M 33: for this galaxy we use the database from Rowe et al. (2005) kindly made available to us. For the $593 \mathrm{C}$ stars in the northern outskirts of M $33\left(\delta>31^{\circ}\right)$, where the internal reddening is expected to be negligible, we obtained $\langle I\rangle=20.15$ and $\langle(V-I)\rangle=2.62$. The reddening towards M 33 is $E(B-V)=$ 0.04 (Schlegel et al. 1998). For a distance of $(m-M)_{0}=24.64$ (Galleti et al. 2004) the above mean magnitude corresponds to $\left\langle M_{I}\right\rangle=-4.57$ and $\left\langle(R-I)_{0}\right\rangle=1.42$ (see Paper I for the colour transformation). This colour is unusually red, we have no explanation for this. The metallicity in this peripheral region of $\mathrm{M} 33$ is $[\mathrm{Fe} / \mathrm{H}]=-1.0$ (Galleti et al. 2004). We evaluate the total number of $\mathrm{C}$ stars in $\mathrm{M} 33$ by estimating the number of $\mathrm{C}$ stars in the truncated southern part of the galaxy, not surveyed by Rowe et al. (2005), by counting those in its corresponding observed northern part. We obtain $8900 \mathrm{C}$ stars, but since there is an obvious incompleteness in the centre we adopt a total population of 10000 stars.

And II: Kerschbaum et al. (2004) identified 7 C stars in this galaxy, however part of And II was certainly outside the observed field and thus this number must be regarded as a lower limit. We used their adopted distance and reddening.

And VII: C star data are from Harbeck et al. (2004). There are no published colours for the $3 \mathrm{CN}$-type stars found and therefore we cannot be certain if these stars meet our colour criterion. The distance and absolute magnitude of this galaxy are from van den Bergh (2000).

Fornax and Leo I: recent spectroscopy by Tolstoy et al. (2003) provides abundance for intermediate-age stars. We adopt $[\mathrm{Fe} / \mathrm{H}]=-1.2$ for Fornax and -1.5 for Leo I. There are no published $I$-magnitudes for the $\mathrm{C}$ stars of Fornax. The number of cool C stars was determined by Demers et al. (2002) from the 2MASS database. Many more $\mathrm{C}$ stars are known in Fornax, most of them located in its crowded centre not efficiently surveyed by $2 \mathrm{MASS}$. The total number of $\mathrm{C}$ stars given in Table 1 for this galaxy thus represents a lower limit.

DDO 190: the data listed in Table 1 for this isolated dwarf galaxy are those given by Battinelli \& Demers (2005b).

\section{Characteristics of the $\mathbf{C}$ star populations}

\subsection{Census}

The relation between the total number of $\mathrm{C}$ stars in a galaxy versus the absolute magnitude of the parent galaxy is displayed in Fig. 1. The strong correlation is somewhat expected and is explained by the small influence of both the star formation history and metallicity of the parent galaxy. It is worth to remind that the apparent total magnitude of a patchy irregular galaxy is often an ill-defined quantity. This is particularly true

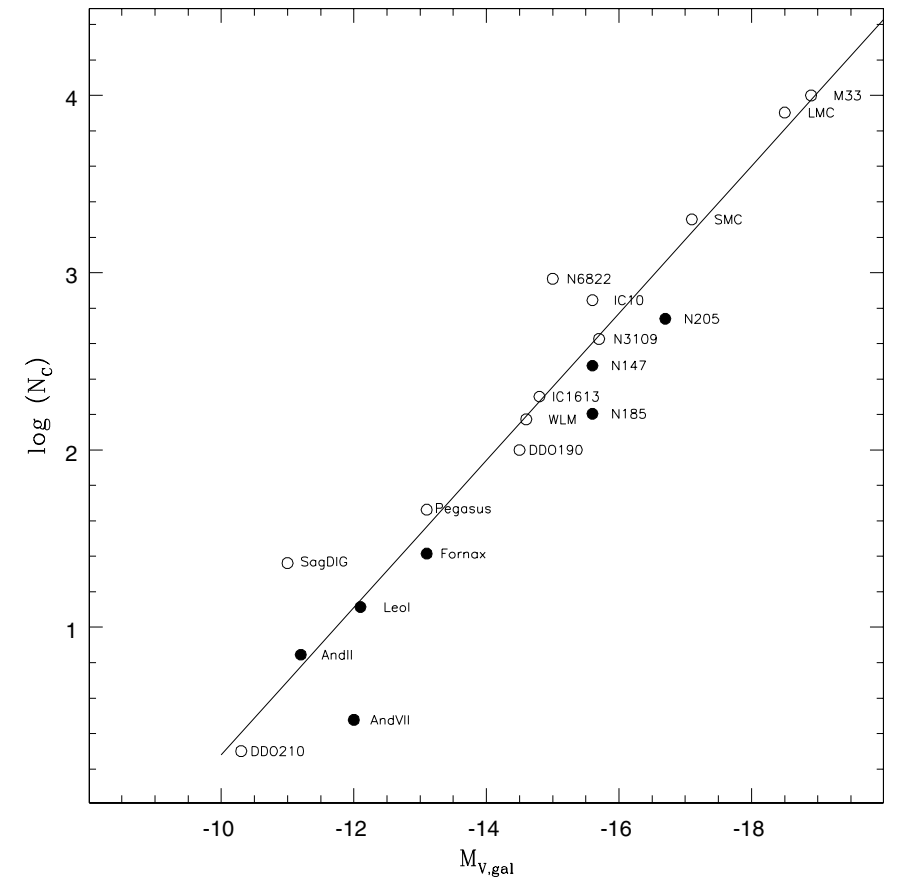

Fig. 1. The logarithm of the number of $\mathrm{C}$ stars versus the total absolute $V$-magnitude of the parent galaxy. The line represents the least-square fit to the points. Filled dots are galaxies with no current star formation.

for galaxies, such as Sag DIG, which are seen behind a dense foreground. An unweighted least-square fit yields

$\log \left(N_{\mathrm{C}}\right)=-4.05-0.425 \times M_{V}$

with a correlation coefficient of $r=0.95$. According to this equation, the $\mathrm{C}$ star population in M31 is expected to be around 90000 stars. Black dots correspond to galaxies with no significant current star formation (ellipticals and spheroidals). They are seen mostly below the line suggesting a deficiency in intermediate-age stars when compared to dwarf irregular galaxies (dIrr's). Dwarf spheroidals, such as Sculptor, Ursa Minor or Carina are not included since the few $\mathrm{C}$ stars discovered in these galaxies are bluer than the $\mathrm{CN}$-type discussed in this paper.

The above relation implies that the specific frequency of $\mathrm{C}$ stars, defined as $\log \left(N_{\mathrm{C}} / L_{\text {Vgal }}\right)=\log \left(N_{\mathrm{C}}\right)+0.4 M_{\text {Vgal }}$, is essentially independent of the absolute luminosity of the parent galaxy, as expected from the theoretical models by Mouhcine $\&$ Lançon (2001). We note however that their predicted value $\log \left(N_{\mathrm{C}} / L_{\mathrm{Vgal}}\right)=-3.0$ is significantly larger than what we found $(\approx-3.9)$. Part of this difference is certainly due to the fact that we are counting only $\mathrm{C}$ stars redder than $(R-I)_{0}=0.9$ but this is far to be enough to match models and observations.

\section{2. $(R-I)$ colours}

Inspection of Table 1 shows that the mean $(R-I)_{0}$ colours of $C$ stars are essentially constant, with the unexplained exception of the large value for M33. No trend with the metallicity of the parent galaxy is seen. In the course of our survey, we noticed two distinct patterns of the colour-colour plot. 


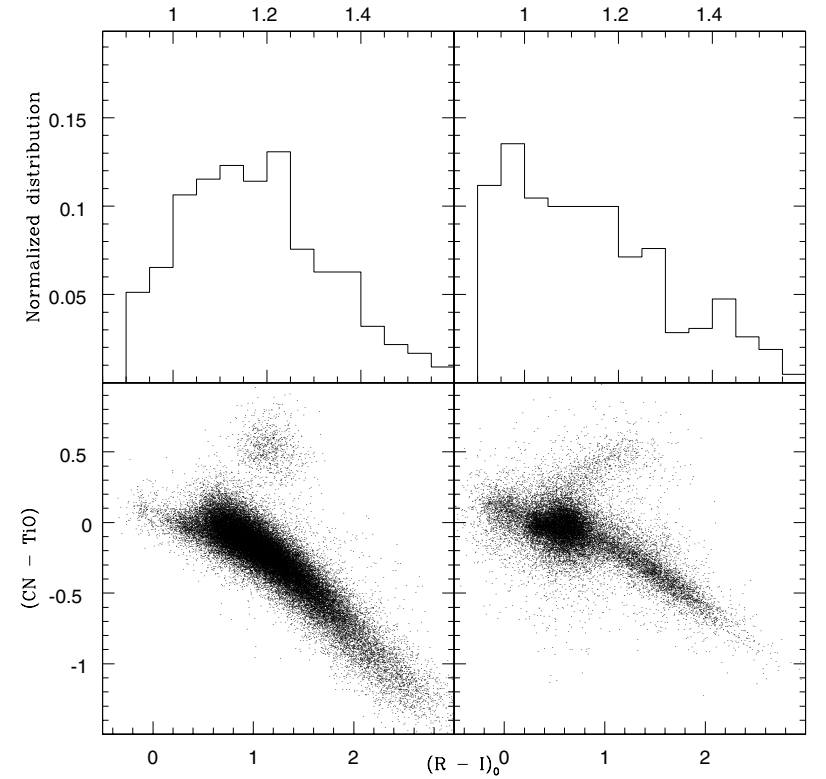

Fig. 2. Two examples of different $\mathrm{C}$ star colour distributions. The left panels represents M 31 and the right ones corresponds to NGC 3109.

The prototype for the first behaviour is M 31 where C stars appear in a clump well separated from the bulk sequence (see Fig. 2). The second behaviour is well illustrated in the colourcolour diagram of NGC 3109 where a continuous branch connects the $\mathrm{C}$ stars to the bulk sequence. This difference is also seen in the $(R-I)_{0}$ colour distribution of $C$ stars, as illustrated in the top panels of Fig. 2. The two galaxies presented in Fig. 2 differ markedly in metallicity, NGC 3109 being more metal poor than M31 and thus the two behaviours could be related to differences in the chemical abundances. However, we found evident counter-examples like e.g. IC 10 (Demers et al. 2004) or IC 1613 (Albert et al. 2000). Unfortunately, no theoretical models have yet attempted to reproduce the observed $(R-I)$ vs. $(\mathrm{CN}-\mathrm{TiO})$ colour diagrams.

\subsection{Absolute magnitudes}

Figure 3 reveals that, for galaxies with at least $100 \mathrm{C}$ stars, the $\left\langle M_{I}\right\rangle$ is essentially constant and equal to $-4.58 \pm 0.11 \mathrm{mag}$. This clearly shows that $\mathrm{C}$ stars can yield distances with uncertainties similar to those of other broadly used distance estimators. Distances to nearby galaxies are reasonably well known from several independent indicators and therefore the scatter observed in Fig. 3 cannot be blamed on distance uncertainties. The scatter greatly increases for faint galaxies and therefore poor statistics are likely to be the major culprit. While galaxies like DDO 210 or And VII (with 2 and 3 C stars, respectively) fit this explanation, Sag DIG does not. In particular, the statistical errors on $\left\langle M_{I}\right\rangle$ are negligible compared to the uncertainties on the distance modulus (typically of the order of $0.1 \mathrm{mag}$ ) and only for And II $( \pm 0.5)$, And VII $( \pm 0.15)$, Leo I $( \pm 0.1)$, SagDIG $( \pm 0.09)$ these errors become important. Sag DIG, not to be confused with the Sagittarius dwarf spheroidal dissolving into the Milky Way, is about $1 \mathrm{Mpc}$ from the Sun. Even though close to the direction of the Galactic centre, the foreground reddening is

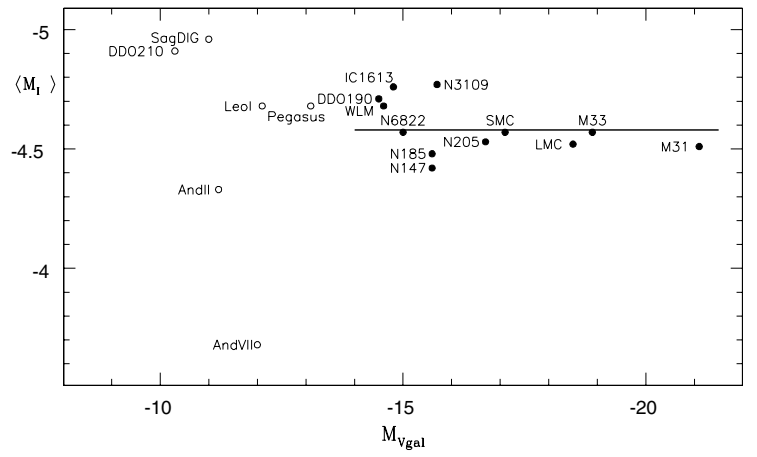

Fig. 3. The mean absolute $I$-magnitude of $\mathrm{C}$ stars is plotted versus the total absolute $V$-magnitude of the parent galaxy. Black dots mark galaxies with over than $100 \mathrm{C}$ stars. The line represents the average magnitude of the black dots.

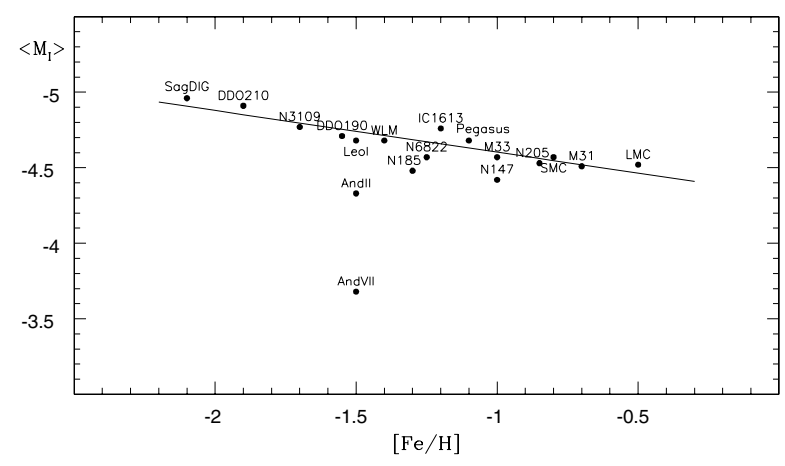

Fig. 4. The mean absolute $I$-magnitude of $\mathrm{C}$ stars versus the adopted metallicity of the parent galaxy (as listed in Table 1). The straight line represents the least-square fit excluding And II and VII.

relatively low and confirmed by several authors (e.g. Momany et al. 2002; Demers \& Battinelli 2002). A second look at this galaxy would be desirable to confirm the lack of fainter $\mathrm{C}$ stars.

A mean magnitude bias introduced by the distance of the galaxy is a possibility that is actually ruled out by our observing technique. Indeed, for the galaxies surveyed we always adjust the exposure times to acquire data with a $\operatorname{good} S / N(20)$ to 1.5 to 2.0 mag below the expected $\langle I\rangle$ of $\mathrm{C}$ stars. Their magnitude distribution is such that this is more than $3 \sigma$ from the mean. The reliability of this approach has recently been confirmed for DDO 190, a galaxy located at $2.8 \mathrm{Mpc}$ where the $\left\langle M_{I}\right\rangle$ of its $67 \mathrm{C}$ stars fits perfectly the $\mathrm{C}$ star populations of Local Group galaxies (Battinelli \& Demers 2005b).

\subsection{Metallicity dependence}

The chemical composition is known to influence the evolution of the AGB stars and in particular the transition from the O-rich to C-rich phase. Metal-poor stars turn sooner, along the AGB, to the C-rich phase and reach brighter luminosity (as a consequence of a less effective mass loss). The net effect of this behaviour on the average $\mathrm{C}$ star luminosity can be evaluated on the basis of complex stellar population models. Mouhcine \& Lançon (2003) models predict the average C star luminosity to be substantially independent of the parent galaxy metallicity. We can now verify such claim. In Fig. 4 the mean absolute 
$I$-magnitude of $\mathrm{C}$ stars is presented as a function of the $[\mathrm{Fe} / \mathrm{H}]$ of the parent galaxies, as listed in Table 1. We see a mild dependence between the two variables and a least-square fit to the points, excluding the outliers And II and And VII, yields:

$\left\langle M_{I}\right\rangle=-4.33+0.28 \times[\mathrm{Fe} / H]$.

Since the publication of Paper I, an homogeneous set of metallicity for 17 Local Group galaxies has been published by McConnachie et al. (2005). Their new results are in fairly good agreement with our adopted values thus do not change the above relation.

\section{Discussion}

As shown in Fig. 3, the mean absolute $I$-magnitude of $\mathrm{C}$ stars can be considered constant for galaxies brighter than $M_{\mathrm{Vgal}}<$ -15 . We note that a certain metallicity dependence of $\left\langle M_{I}\right\rangle$ is detected (Fig. 4). This introduces an evident complication in the straightforward application of $\mathrm{C}$ stars for distance estimates. However, when galaxies with $\mathrm{Fe} / \mathrm{H}]>-1.5$ are considered, the $\left\langle M_{I}\right\rangle$ is fairly constant with an average of $-4.57 \pm 0.10$, thus with an uncertainty comparable to the typical errors of other secondary distance indicators. Therefore, the $\left\langle M_{I}\right\rangle$ of $\mathrm{C}$ stars can be in principle used to determine the distances of spiral and elliptical galaxies which fulfill the two requirements: 1) are not metal-poor systems; 2) are large enough to host a numerous population of $\mathrm{C}$ stars.

Presently, the C star approach is hinder by the fact that nearly all the big galaxies in the neighbourhood of the Local Group already have well established distances. Indeed, to reach more than $2 \mathrm{Mpc}$, the use of narrow band filters with current ground-based telescopes requires prohibitively long exposures. Other broad-band identification techniques could in principle help to push this method to larger distances. The SLOAN $\left(r^{\prime}-i^{\prime}\right)$ vs. $\left(g^{\prime}-r^{\prime}\right)$ has been exploited by Margon et al. (2002) and Downes et al. (2004) to identify numerous C stars in the Galactic halo. Unfortunately, there is a huge population of quasars, with $z>3$ and $i^{\prime}>19$, which overlaps with $\mathrm{C}$ stars in the colour-colour diagram (Demers \& Battinelli, in preparation) and thus a background contamination has to be taken into account.

$\mathrm{C}$ stars can be easily identified from their near-IR colours. Weinberg \& Nikolaev (2001) have shown that $C$ stars, selected in a narrow colour range in the $J, K_{\mathrm{S}} \mathrm{CMD}$, are potential standard candles since their $K_{\mathrm{s}}$ magnitude spread is quite small. This near-IR approach has been shown by Demers et al. (2002) to be equivalent to the $\mathrm{CN}$, TiO method. $\mathrm{C}$ stars have $M_{K_{\mathrm{s}}}$ magnitudes around $-7.7 \mathrm{mag}$ but the constancy of $\left\langle M_{K_{\mathrm{s}}}\right\rangle$ is not yet fully proved. The average $M_{K_{\mathrm{s}}}$ of $\mathrm{C}$ stars, identified in the range $1.4<\left(J-K_{\mathrm{s}}\right)<2.0$, is known - from somewhat inhomogeneous sources - only for: LMC and Fornax (2MASS), SMC (DENIS) and NGC 6822 (Cioni \& Habing 2005). The $\left\langle M_{K_{\mathrm{s}}}\right\rangle$ for these galaxies show $\sim 0.4$ mag spread. Obviously, a larger and homogeneous sample of galaxies with various luminosities and chemical compositions would help to better assess this point. Near infrared $J, K_{\mathrm{S}}$ filters are universally available on the major telescopes. Moreover, wide-field imagers, as WIRCAM at the CFHT or WFCAM at the UKIRT, are now fully operative. Unfortunately, the bright night sky in the $K_{\mathrm{s}}$ band implies exposure times which do not represent a significant improvement over the $\mathrm{CN}, \mathrm{TiO}$ technique. A major improvement of the near infrared technique could be achieved by space-based observations. In the JWST era C stars, being brighter than RGB stars, could represent an advantageous approach to distance determinations thus complementing the TRGB method.

Acknowledgements. This research is funded in part (S.D.) by the Natural Sciences and Engineering Research Council of Canada.

\section{References}

Albert, L., Demers, S., \& Kunkel, W. E. 2000, AJ, 119, 2780 Bellazzini, M., Ferraro, F. R., \& Pancino, E. 2001, AJ, 556, 635 Battinelli, P., \& Demers, S. 2005a, A\&A, 434, 657 (Paper I) Battinelli, P., \& Demers, S. 2005b, A\&A, submitted Battinelli, P., Demers, S., \& Letarte, B. 2003, AJ, 125, 1298 Cioni, M.-R. L., \& Habing, H. J. 2003, A\&A, 402, 133

Cioni, M.-R. L., \& Habing, H. J. 2005, A\&A, 429, 837

Cook, K. H., Aaronson, M., \& Norris, J. 1986, ApJ, 305, 634

Corradi, R. L. M., Magrini, L., Greimel, R., et al. 2005, A\&A, 431, 555

Costa, E., \& Frogel, J. A. 1996, AJ, 112, 2607

Demers, S., \& Battinelli, P. 2002, AJ, 123, 238

Demers, S., Dallaire, M., \& Battinelli, P. 2002, AJ, 123, 3428

Demers, S., Battinelli, P., \& Letarte, B. 2004, A\&A, 424, 125

Downes, R. A., Margon, B., Anderson, S., et al. 2004, AJ, 127, 2838

Galleti, S., Bellazzini, M., \& Ferraro, F. R. 2004, A\&A, 423, 925

Graham, J. A. 1982, ApJ, 252, 474

Harbeck, D., Gallagher, J. S., \& Grebel, E. K. 2004, AJ, 127, 2711

Jacoby, G. H., Branch, D., Ciardullo, R., et al. 1992, PASP, 104, 599

Kerschbaum, F., Nowotny, W., Olofsson, H., \& Schwarz, H. E. 2004, A\&A, 427, 613

Lee, M. G., Freedman, W. L., \& Madore, B. F. 1993, ApJ, 417, 553

Letarte, B., Demers, S., Battinelli, P., \& Kunkel, W. E. 2002, AJ, 123, 832

Margon, B., Anderson, S. F., Harris, H. C., et al. 2002, AJ, 124, 1651

McConnachie, A., Irwin, M., Ibata, R., et al. 2005, MNRAS, 356, 979

Momany, Y., Held, E. V., Saviane, I., \& Rizzi, L. 2002, A\&A, 384, 393

Mouhcine, M., \& Lançon, A. 2003, MNRAS, 338, 572

Nikolaev, S., \& Weinberg, M. D. 2000, ApJ, 542, 804

Palmer, L. G., \& Wing, R. F. 1982, AJ, 87, 1739

Pritchet, C. J., Richer, H. B., Schade, D., Crabtree, D., \& Yee, H. K. C. 1987, ApJ, 323, 79

Richer, H. B., Crabtree D. R., \& Pritchet, C. J. 1984, ApJ, 287, 138

Richer, H. B., Pritchet, C. J., \& Crabtree, D. R., 1985, ApJ, 298, 240

Rowe, J., Richer, H. B., Brewer, J. P., \& Crabtree, D. R. 2005, AJ, 129, 729

Schlegel, D., Finkbeiner, D., \& Davis, M. 1998, ApJ, 500, 525

Tolstoy, E., Venn, K. A., Shetrone, M., et al. 2003, AJ, 125, 707

van den Bergh, S. 2000, The Galaxies of the Local Group, Cambridge Astrophysics Ser., 35

Weinberg, M. D., \& Nikolaev, S. 2001, ApJ, 548, 712 